\title{
Optimal partitioning of a square: a numerical approach
}

\author{
Supanut Chaidee ${ }^{\mathrm{a}, \mathrm{b}}$, Wacharin Wichiramala ${ }^{\mathrm{b}, *}$ \\ a Graduate School of Advanced Mathematical Sciences, Meiji University, 4-21-1 Nakano, Nakano-ku, \\ Tokyo 164-8525, Japan \\ b Department of Mathematics and Computer Science, Faculty of Science, Chulalongkorn University, \\ 254 Phayathai Road, Pathumwan, Bangkok 10330 Thailand
}

*Corresponding author, e-mail: wacharin.w@chula.ac.th

Received 9 Mar 2015

Accepted 11 Apr 2016

\begin{abstract}
Optimal partitioning of a square is the search for the least-diameter way to partition a unit square into $n$ pieces. The problem is here solved for some small $n$ values. Although this problem has recently been approached by transforming the problem into a graphical enumeration, the algorithm had too large a computational cost for cases of $n \geqslant 7$. In this paper, the existence of solutions in a more general sense is established and the graphical transformation method is improved by generating dual graphs of the combinatorial patterns. In particular, combinatorial patterns were generated using the triangulation of planar graphs. Theorems to eliminate some unnecessary partitions are presented and numerical optimization by convex programming is used to find the minimum diameters. Our results confirm the earlier reported cases for $n=9$ and 10 and the predictions made for the case of $n=11$.
\end{abstract}

KEYWORDS: square partitioning, least diameter

MSC2010: 52A40 52-04

\section{INTRODUCTION}

The optimal partitioning of a square is the search for the least-diameter way to partition a unit square into $n$ pieces. The problem was first solved for the case of $n=3$ in 1958 after being formulated as the following problem by Wenceslas and Lipman ${ }^{1}$. Prove that a unit square which is dissected by a $\frac{1}{8} \times 1$ rectangular piece and two $\frac{1}{2} \times \frac{7}{8}$ rectangular pieces cannot be covered by three sets having diameters less than $d=\sqrt{65} / 8$. A similar problem in the case of $n=5$ was posed in 1959 by Page ${ }^{2}$ and later solved in Ref. 3. The problem was extended from the case $n=3$ to find the minimum $d$ such that the square is covered by five sets each of which has diameter $d$.

Graham $^{4}$ studied a similar problem on the partitioning of an equilateral triangle into $n$ pieces for $n$ up to 15 , while the most significant process on the unit square was performed by Guy and Selfridge ${ }^{5}$. In brief, they defined the optimal diameter of partitioning the unit square into $n$ pieces by the infimum among all possible patterns of the maximum of a polygonal diameter of an $n$-piece partition. They found and proved the exact values of the optimal diameters for the cases of $n=4$ to 10 , and gave numerical bounds and a prediction for cases of a larger $n$. Unfortunately, the proof for each $n$ has its own unique technique with no relation to the proof for the other cases. Later, Jepsen ${ }^{6}$ worked independently to prove the cases up to $n=6$.

In our recent work ${ }^{7}$, the optimal partitioning of a square from a new viewpoint of transforming the problem into graphical enumeration was proposed along with the algorithm to generate all the possible patterns and optimize them in the case $n=4$ to 7 , along with the numerical optimal diameter. However, for larger $n$ values the algorithm is too computationally costly.

In this paper, we present an algorithm to find the numerical optimal diameter, the results from which were found to agree with the previous work of Guy and Selfridge ${ }^{5}$ up to the 6th decimal place for $n$ values up to 11 . This paper is organized as follows. In the first section, we establish the existence of a solution in a more general sense and then review the problem transformation from a geometric problem to a graphical enumeration and give an overview of the process. We then propose the algorithm that relates the dual graphs and their triangulation and provide the necessary condition of optimal partition which can eliminate some unnecessary patterns during the pattern enumeration process. The enumeration for $n=9$ to 11 is also included in this part. Numerical results and patterns 
for the cases of $n=9$ to 11 are given in the final section.

\section{PROBLEM FORMULATION}

Throughout this paper, we denote $S$ as a unit square in $\mathbb{R}^{2}$. For any set $T$ in $\mathbb{R}^{2}$, we define the diameter of $T$ by $\operatorname{diam}(T)=\sup \{d(p, q) \mid p, q \in T\}$, where $d(p, q)$ is the Euclidean distance between $p$ and $q$.

\section{Existence of the solution}

To prove the existence of the solution, the problem was evaluated in a more general sense as follows. The original problem asks for the diameter optimizing partition $\left\{S_{i}\right\}$ of a closed unit square in $\mathbb{R}^{2}$, and the authors show the existence of such optimizing partitions and that each one may be considered to be composed of convex polygons ${ }^{5}$. In this work, we allow $S_{i}^{\prime} s$ to overlap and then show later that it suffices to consider only convex polygonal $S_{i}^{\prime} s$ with disjoint interiors.

Since $\operatorname{diam}(C)=\operatorname{diam}(\bar{C})$, where $\bar{C}$ is a closure of $C$ in $\mathbb{R}^{2}$, it is necessary to focus on covering by closed sets as in the following definition.

Definition 1 Let $S$ be a unit square and $C_{i} \subseteq S$ for $i=1,2, \ldots, n$ be closed subsets. $\mathscr{C}=\left\{C_{1}, C_{2}, \ldots, C_{n}\right\}$ is called the closed covering set of a square $S$ if $\bigcup_{i=1}^{n} C_{i}=S$.

Theorem 1 shows that the solutions to the generalized problem are still virtually unchanged.

Theorem 1 For a given natural number n, there exists a closed covering with the minimum diameter of the square.

Proof: Let $\mathfrak{C}$ be a family of closed covering sets of a square. For $\mathscr{C} \in \mathfrak{C}$, such that $\mathscr{C}=\left\{C_{1}, C_{2}, \ldots, C_{n}\right\}$, we define $\operatorname{Diam}(\mathscr{C})=\max _{i \in\{1,2, \ldots, n\}} \operatorname{diam}\left(C_{i}\right)$. Since $\operatorname{Diam}(\mathscr{C}) \geqslant 0$, there exists inf $\mathscr{C}_{\mathscr{C} \in \mathfrak{C}} \operatorname{Diam}(\mathscr{C})$, denoted by $\alpha$. Next, we will find $\mathscr{C}_{0}$ with diameter $\alpha$.

When $\alpha \in\{\operatorname{Diam}(\mathscr{C}) \mid \mathscr{C} \in \mathfrak{C}\}$ it is clear that there exists $\mathscr{C}_{0}$ such that $\operatorname{Diam}\left(\mathscr{C}_{0}\right)=\alpha$.

When $\alpha \notin\{\operatorname{Diam}(\mathscr{C}) \mid \mathscr{C} \in \mathfrak{C}\}$ there is a sequence of covers $\left\{\mathscr{C}_{k}\right\}$ such that $\alpha=\lim _{k \rightarrow \infty} \operatorname{Diam}\left(\mathscr{C}_{k}\right) . \quad$ By the compactness argument under the Hausdorff metric, there exists a subsequence $\left\{C_{k, m}\right\}_{m=1}^{\infty}$ converging to a covering set $\mathscr{C}$ in $\mathfrak{C}$. Clearly, $\operatorname{diam}(\mathscr{C})=\alpha$, a contradiction. Hence this case is impossible. Hence there exists a closed covering of a square with a minimum diameter.

Theorem 1 shows that we can cover the square with any closed sets. However, it suffices to cover a square by convex sets by the following arguments.
Let $\mathscr{H}\left(C_{i}\right)$ be the convex hull of $C_{i}$. Note that $\operatorname{diam}\left(C_{i}\right)=\operatorname{diam}\left(\mathscr{H}\left(C_{i}\right)\right)$, and for any convex set $K, \operatorname{diam}(K)=\operatorname{diam}(\bar{K})$. Hence we can focus on the closed convex $C_{i}^{\prime} s$. In this case, $C_{i}$ may be overlapped by the adjacent set $C_{j}$. If the interior of $C_{i}$ and $C_{j}$ are not disjoint, it is possible to construct a cover of a smaller diameter by replacing $C_{i}$ by

$$
\overline{C_{i} \backslash \bigcup_{j=1}^{i-1} C_{j}}
$$

When any two closed convex sets have disjoint interiors, they may overlap only at their boundaries. By convexity, the overlapped boundaries should be straight lines.

By the previous arguments, we can consider the covering problem with partitioning a square into convex polygonal pieces, which is similar to the original problem by Guy and Selfridge ${ }^{5}$.

We define the optimal diameter to be consistent to the previous studies ${ }^{5}$. The rigorous definition of optimal diameter and numerical optimal diameter is defined as follows.

Definition 2 For a closed unit square $S$, we denote the family $\Pi$ of all partition $\pi$ of $S$ into $n$ disjoint subset $S_{i}$ such that $\cup S_{i}=S$. The optimal diameter $d_{n}$ is defined by

$$
d_{n}=\inf _{\pi \in \Pi} \max _{1 \leqslant i \leqslant n} \sup _{P, Q \in S_{i}} d(P, Q),
$$

where $P$ and $Q$ are points in $S_{i}$, and $d(P, Q)$ is the Euclidean distance between $P$ and $Q$. Such $\pi \in \Pi$ attaining the optimal diameter is said to be the optimal partition.

We denote the optimal diameter numerically computed from the frameworks in the previous study ${ }^{7}$ and in this paper by $D_{n}$.

\section{Problem transformation}

Based upon the original problem by Guy and Selfridge $^{5}$, we consider each partition as an embedded planar graph with straight edges. Our main concern is to generate all the possible graphs satisfying the condition of being necessary optimal partitions. Furthermore, we found that it suffices to consider only the graphs with vertices of degree 3 . This is because each vertex of a degree greater than three may be viewed as a bunch of vertices of degree 3 with extra edges of length zero, while a vertex of degree 2 can be ignored since each face is convex.

We define the generation of the graph of degree 3 vertices corresponding to the partitioning of the square as follows. 
Definition 3 A straight line planar graph $\pi$ is said to be a combinatorial pattern if $\pi$ satisfies: (i) $\pi$ is a 3-regular graph, (ii) $\pi$ does not have a loop or multiple edges, (iii) the internal edges form a connected graph.

If $\pi_{1}$ and $\pi_{2}$ are combinatorial patterns, then $\pi_{1}$ and $\pi_{2}$ are distinct if they are not isomorphic.

The overview process for transforming a problem has been presented in our previous work ${ }^{7}$, and in brief is as follows.

(i) Construct a condition to enumerate the number of vertices and edges by Euler's formula and the condition of 3-regular graphs.

(ii) Join the vertices with edges of every possible case that leads to a combinatorial pattern.

(iii) Optimize each combinatorial pattern.

\section{Notation}

Let $S$ be a unit square on the plane; $\pi$ be a combinatorial pattern; and $\mathscr{V}, \mathscr{E}$ and $\mathscr{F}$ be the set of vertices, edges and faces, respectively. Let $\mathscr{V}_{1}, \mathscr{V}_{2}, \mathscr{V}_{3}$ and $\mathscr{V}_{4}$ be the set of vertices on the top, left, bottom and right sides of the square, respectively; and similarly for $\mathscr{F}_{i}$. Let $\mathscr{V}_{0}$ and $\mathscr{F}_{0}$ be the set of vertices and faces that are not adjacent to any side of the square. The cardinality of $\mathscr{V}_{i}$ and $\mathscr{F}_{i}$ are denoted by $V_{i}$ and $F_{i}$.

For faces in $\mathscr{F}_{1}$, the faces are designated from the upper right corner proceeding anticlockwise by $f_{11}, f_{12}, \ldots, f_{1 F_{1}}$. They are designated by starting at the upper left corner in the same manner by $f_{21}, f_{22}, \ldots, f_{2 F_{1}}$. The third and fourth sides are designated similarly. We also obtain $f_{1 F_{1}}=f_{21}$, $f_{2 F_{2}}=f_{31}, f_{3 F_{2}}=f_{41}, f_{4 F_{4}}=f_{11}$. A face in the graph $\pi$ is considered as a vertex in the dual graph $\pi^{*}$. The notation $f_{i j}$ also represents a vertex in $\pi^{*}$. If a face $f_{i}$ is adjacent to $f_{j}$, we write $f_{i} \simeq f_{j}$, otherwise $f_{i} \nsucceq f_{j}$. In the dual graph construction, if $f_{i}$ connects to $f_{j}$, then $f_{i} \sim f_{j}$, otherwise $f_{i} \not f_{j}$. Fig. 1 illustrates the usage of provided notation.

\section{THE PROPOSED ALGORITHM}

Definition 4 Let $\pi$ be a combinatorial pattern. The dual graph $\pi^{*}$ of $\pi$ is called the dual combinatorial pattern.

\section{Triangulation process}

Suppose that $\pi$ is a combinatorial pattern. Since $\pi$ is a 3-regular graph, the dual graph $\pi^{*}$ of $\pi$ is a planar graph composed of triangular faces. Hence we need to triangulate a set of vertices of a dual graph $\pi^{*}$.

Let $\mathscr{F}_{\text {out }}$ be a sequence of outer vertices, $\mathscr{F}_{\text {in }}$ be a set of interior vertices, $\mathscr{F}_{\triangle}$ be a set of current

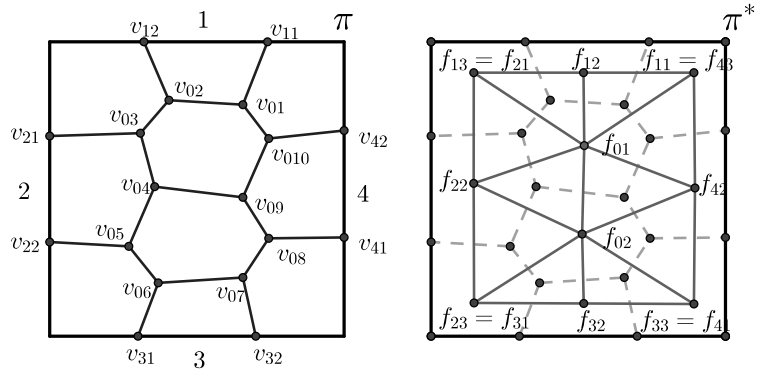

Fig. 1 An example of a pattern $\pi$ (left) and its dual graph $\pi^{*}$ (right). In this example, $\mathscr{V}_{1}=\left\{v_{11}, v_{12}\right\}, \mathscr{V}_{2}=\left\{v_{21}, v_{22}\right\}$, $\mathscr{V}_{3}=\left\{v_{31}, v_{32}\right\}, \mathscr{V}_{4}=\left\{v_{41}, v_{42}\right\}, \mathscr{V}_{0}=\left\{v_{01}, \ldots, v_{010}\right\}$ and $f_{11} \simeq f_{12}, f_{11} \simeq f_{42}, f_{11} \simeq f_{01}$ but $f_{11} \not f_{02}$. The numbers 1-4 in the left panel represent the side of the square.

triangles initially set to be empty, and $\mathscr{P}$ be a set of prohibiting connections.

The following algorithm is a recursive process:

(i) Join the vertices $\mathscr{F}_{\text {out }}$ sequentially.

(ii) Triangulation Process: Consider $\mathscr{F}_{\text {in }}$ and $\mathscr{F}_{\text {out }}$.

A. If $\mathscr{F}_{\text {in }} \neq \varnothing$, then

1. construct a triangle whose vertices are from two consecutive vertices $\left(f_{\alpha} f_{\beta}\right)$ in a sequence $\mathscr{F}_{\text {out }}$ and a vertex $f_{\gamma}$ in $\mathscr{F}_{\text {in }}$;

2. add a triangle $f_{\alpha} f_{\beta} f_{\gamma}$ to $\mathscr{F}_{\Delta}$, delete $f_{\gamma}$ from $\mathscr{F}_{\text {in }}$, and add edge $f_{\alpha} f_{\beta}$ to $\mathscr{P}$;

3. insert $f_{\beta}$ between $f_{\alpha}$ and $f_{\beta}$ in $\mathscr{F}_{\text {out }}$;

4. go back to (ii).

B. If $\left|\mathscr{F}_{\text {out }}\right| \geqslant 3$, then

1. construct a triangle by three consecutive vertices $f_{\text {il }}, f_{j m}, f_{k p}$ in $\mathscr{F}_{\text {out }}$ that satisfy at least one of the following conditions: $i \neq j$ or $j \neq k$ or $i \neq k$;

2. add a triangle $f_{\text {il }} f_{j m} f_{k p}$ to $\mathscr{F}_{\triangle}$;

3. delete $f_{j m}$ from $\mathscr{F}_{\text {out }}$;

4. go back to (ii).

(iii) If $\left|\mathscr{F}_{\text {out }}\right|=3$ and $\mathscr{F}_{\text {in }}=\varnothing$, then the process is complete.

We note that the condition of $\mathscr{P}$ will be controlled in every step of the triangulation process. The prohibited condition will be provided in the next section. Fig. 2 illustrates the mentioned algorithm.

The main process of this work is to generate all the possible dual combinatorial patterns. After all the dual combinatorial patterns are obtained, they are converted back to the respective combinatorial patterns and each pattern is optimized. To clarify, the overall process is summarized in the following 
(a)

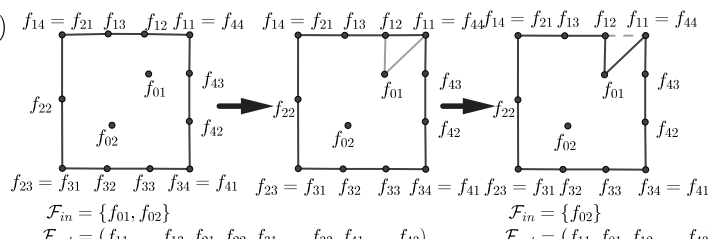

b) $f_{14}=f_{21} f_{13} f_{12} f_{11}=f_{44} \quad f_{14}=f_{21} f_{13} \quad f_{12} f_{11}=f_{44} f_{14}=f_{21} f_{13} \quad f_{12} \quad f_{11}=f_{44}$

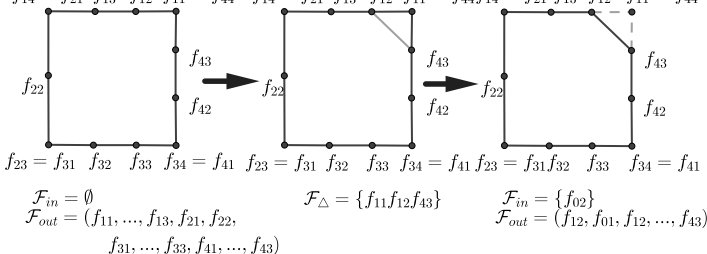

Fig. 2 Illustration of the triangulation algorithm: (a) $\mathscr{F}_{\text {in }} \neq \varnothing$; (b) $\left|\mathscr{F}_{\text {out }}\right| \geqslant 3$.

steps.

(i) Consider the number of faces on each side by doing an integer partition. To clarify this, we find the possible integer solutions of

$$
F_{0}+F_{1}+F_{2}+F_{3}+F_{4}=n+4,
$$

where the condition of $F_{0}, \ldots, F_{4}$ is determined in the next section. The solution of (2) is written as $\left(F_{0}, F_{1}, F_{2}, F_{3}, F_{4}\right)$. By fixing $F_{0}$, the following solutions are the same by rotating and flipping the square: $\left(F_{0}, F_{1}, F_{2}, F_{3}, F_{4}\right)=\left(F_{0}, F_{2}, F_{3}, F_{4}, F_{1}\right)=$ $\left(F_{0}, F_{3}, F_{4}, F_{1}, F_{2}\right)=\left(F_{0}, F_{4}, F_{1}, F_{2}, F_{3}\right)$, and $\left(F_{0}, F_{1}, F_{2}, F_{3}, F_{4}\right)=\left(F_{0}, F_{3}, F_{2}, F_{1}, F_{4}\right)=$ $\left(F_{0}, F_{2}, F_{1}, F_{4}, F_{3}\right)=\left(F_{0}, F_{1}, F_{4}, F_{3}, F_{2}\right)$.

(ii) For each solution $\left(F_{0}, F_{1}, F_{2}, F_{3}, F_{4}\right)$ from (i), choose a representation of each face to be a vertex in the dual graph, and consider a set of $\mathscr{F}_{\text {out }}, \mathscr{F}_{\text {in }}, \mathscr{F}_{\triangle}$ and $\mathscr{F}_{\text {proh }}$.

(iii) Triangulate a set $\mathscr{F}_{\text {in }} \cup \mathscr{F}_{\text {out }}$ by the stated algorithm. The results are called dual combinatorial patterns. Each prohibited connection will be denoted by an element $\left\{f_{i k}, f_{\mathrm{j} l}\right\}$ in $\mathscr{P}$ if $f_{i k} \sim f_{\mathrm{j} 1}$ is not allowed.

(iv) Convert the dual combinatorial patterns to combinatorial patterns.

(v) Optimize each combinatorial pattern.

To speed up the generating process, and so avoid generating isomorphic patterns, the symmetrical patterns were considered as follows.

Definition 5 Let $T=\left(F_{0}, F_{1}, F_{2}, F_{3}, F_{4}\right)$ be a solution of (2). $T$ is symmetric with respect to $X$-axis if $F_{1}=F_{3} . \quad T$ is symmetric with respect to $Y$-axis if $F_{2}=F_{4} . T$ is diagonal symmetric if $F_{1}=F_{2}$ and $F_{3}=F_{4}$ or $F_{1}=F_{4}$ and $F_{2}=F_{3}$.
We conclude that if $T_{1}$ is symmetric with respect to $T_{2}$, the graphs generated from $T_{1}$ will be isomorphic to those generated from $T_{2}$.

\section{Optimization process}

For each vertex $(P, Q)$ in a combinatorial pattern, we denote the coordinates by $P\left(x_{i}, y_{i}\right)$ and $Q\left(x_{j}, y_{j}\right)$ and define

$$
f\left(x_{i}, y_{i}, x_{j}, y_{j}\right)=\sqrt{\left(x_{i}-x_{j}\right)^{2}+\left(y_{i}-y_{j}\right)^{2}} .
$$

Then $f$ is a convex function. When the domain of $f$ is convex, minimizing $f$ is of the type called convex programming. For each face in a combinatorial pattern, the maximum $\max f_{k}\left(x_{i}, y_{i}, x_{j}, y_{j}\right), \pi \in \Pi$ over all possible pairs of vertices is also convex. Hence the maximum $\left(d_{\max }\right)$ of these $\max f_{k}\left(x_{i}, y_{i}, x_{j}, y_{i}\right)$ over all faces of $\pi$ is convex.

\section{METHODS TO ELIMINATE THE UNNECESSARY PARTITIONS}

Although the proposed algorithm can generate all the possible combinatorial patterns, some of the generated patterns do not turn out to be the optimal partition. Those patterns are called unnecessary partitions. In this section, we provide the necessary conditions of a combinatorial pattern to be the optimal partition, which can help to eliminate the unnecessary combinatorial patterns. Note that the lemmas and theorems were constructed using the proved results of an earlier report by Guy and Selfridge ${ }^{5}$.

\section{Theorems to eliminate unnecessary partitions}

For a unit square $S$, we define $A, B, C$ and $D$ as vertices of the unit square, starting from the lower left corner and going anticlockwise. Hence $A$ is at $(0,0)$.

Lemma 1 (Ref. 7) A subdivided partition has a smaller or equal diameter than the original partition.

By Lemma $1, d_{n}$ is a non-increasing sequence. The following lemma and theorems show the necessary condition of being an optimal partition or combinatorial pattern.

Lemma 2 For $n \geqslant 4$, if the optimal partition has the combinatorial pattern $\pi$, then $\pi$ has at least one vertex on each side.

Proof: Suppose that there is a side which does not contain a vertex. Without loss of generality, assume that the side is $\overline{A D}$. Since $|\overline{A D}|=1$, a piece containing $\overline{A D}$ has diameter of at least 1 . However, $d_{4}=\sqrt{2} / 2<1$, a contradiction. 


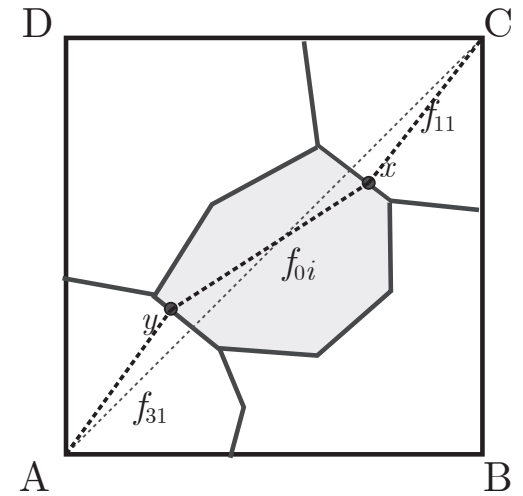

Fig. 3 The situation $f_{11} \simeq f_{0 i} \simeq f_{31}$.

Theorem 2 For $n \geqslant 9$, if the optimal partition has the combinatorial pattern $\pi$, then (i) $\pi$ has at least two vertices on each side and (ii) $\pi$ has at lease one interior piece.

Proof: (i) The proof is similar to that in Lemma 2 but using $d_{9}=5 / 11$. (ii) Suppose that $\pi$ has no interior piece. Choose a point $R\left(\frac{1}{2}, \frac{1}{2}\right)$ in the square $S$. Hence there is a piece $f_{i j}$. Since $f_{i j}$ is placed on the $i$ th side, $d(R, X) \geqslant 1 / 2>d_{9}$ such that $X$ is a point on the $i$ th side that belongs to $f_{i j}$. Thus $\pi$ does not give the optimal diameter.

Theorem 3 (Ref. 7) For $n \geqslant 4$, the combinatorial pattern $\pi$ of an optimal partition may not satisfy either of the following conditions: (i) there is no edge from $\mathscr{V}_{i}$ to $\mathscr{V}_{j}$ for $i \neq j$; (ii) there is no interior vertex incident to two vertices from opposite boundaries.

We denote a set satisfying Theorem 3(i) by $\mathscr{O}$, which we put in the set $\mathscr{P}$.

Theorem 4 For $n \geqslant 9$, the combinatorial pattern $\pi$ of an optimal partition may not satisfy the conditions: (i) there are two adjacent faces from opposite boundaries; (ii) there is no face adjacent to both $f_{11}$ and $f_{31}$ or both $f_{21}$ and $f_{41}$.

Proof: (i) Without loss of generality, suppose that there is a face in $\mathscr{F}_{1}$ and a face in $\mathscr{F}_{3}$ that are adjacent. Denote a point on the first side which belongs to $f_{1 i}$ and a point on the third side which belongs to $f_{3 j}$ as $v_{1}$ and $v_{2}$, respectively. Hence there is $x \in f_{1 i} \cap f_{3 j}$ such that $d\left(v_{1}, x\right)+d\left(x, v_{2}\right) \geqslant 1$. Hence the length of segment $\overline{v_{1} x}$ or $\overline{x v_{2}}$ should be greater or equal to $1 / 2$ which means that $d_{9}=5 / 11<1 / 2$.

(ii) Suppose that $f_{0 i}$ is a face satisfying $f_{11} \simeq$ $f_{0 i} \simeq f_{31}$. Note that $f_{11}$ and $f_{31}$ are laid on the corners of a square which is shown in Fig. 3 . The
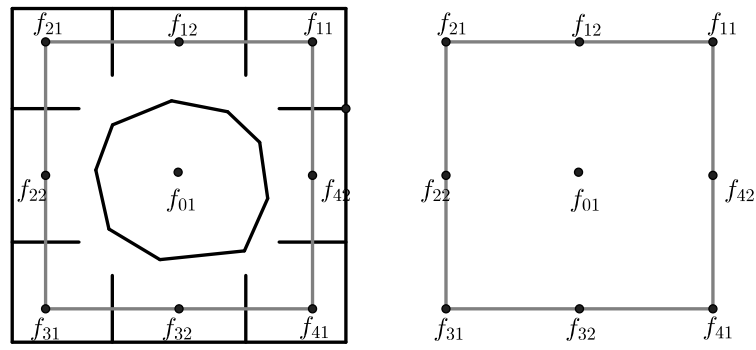

Fig. 4 The initial combinatorial pattern of the solution $(1,3,3,3,3)$ (left); the corresponding initial triangulating data (right).

points $A$ and $C$ are on the boundary of the square which is the vertex of $f_{31}$ and $f_{11}$, respectively. We note that $d(A, C)=\sqrt{2}$. By the assumption that $f_{11} \simeq f_{01} \simeq f_{31}$ and the segment $\overline{A C}$ meets $f_{11}, f_{01}$ and $f_{31}$, there are $x \in f_{11} \cap f_{01}$ and $y \in f_{31} \cap f_{01}$ such that $d(C, x)+d(x, y)+d(y, A)>d(A, C)=\sqrt{2}$. Hence one of the segments $\overline{C x}, \overline{x y}$, or $\overline{y A}$ has length greater or equal to $\sqrt{2} / 3$. This contradicts that $d_{9}=5 / 11<\sqrt{2} / 3$.

Hence we may define a partition or a combinatorial pattern to be unnecessary if it does not satisfy Lemma 2, Theorem 2, Theorem 3, or Theorem 4.

\section{COMBINATORIAL PATTERNS ENUMERATION}

To enumerate combinatorial patterns efficiently, we do not generate some of unnecessary patterns as they do not lead to optimal partitions. We put those unnecessary conditions in $\mathscr{P}$. We enumerate patterns for $n=9$ to 11 as follows.

\section{Enumeration of the case $\boldsymbol{n}=\mathbf{9}$}

Equation (2) is written in the form

$$
F_{0}+F_{1}+F_{2}+F_{3}+F_{4}=13 .
$$

From Theorem 2, we have the conditions $F_{0} \geqslant 1$ and $F_{1}, F_{2}, F_{3}, F_{4} \geqslant 3$. Hence the only solution of (3) is $(1,3,3,3,3)$. We consider the initial triangulation data in Fig. 4.

From Theorem 4, we must avoid the situation $f_{11} \sim f_{01} \sim f_{31}$ and $f_{21} \sim f_{01} \sim f_{41}$. Using a treediagram and the symmetry, the only condition we have is $\left(f_{01} \not f_{11}\right) \wedge\left(f_{01} \not f_{21}\right)$.

Since the solution $(1,3,3,3,3)$ is symmetric with respect to the $X$-axis and $Y$-axis and is also diagonally symmetric, it suffices to consider the condition $\left(f_{01} \not f_{11}\right) \wedge\left(f_{01} \not f_{21}\right)$ together with the condition from Theorem 3 . Hence we have to 

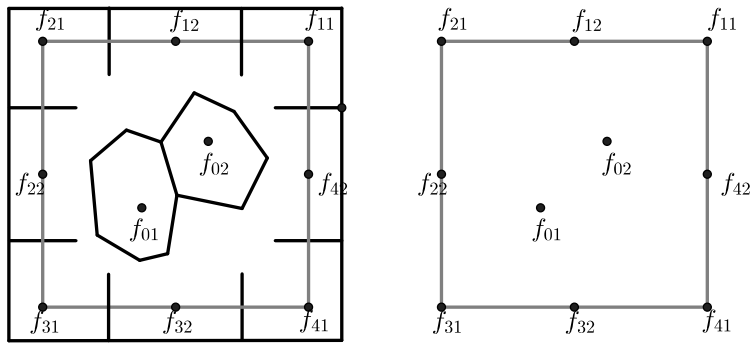

Fig. 5 The initial combinatorial pattern of the solution $(2,3,3,3,3)$ (left); the corresponding initial triangulating data (right).

triangulate:

$$
\begin{aligned}
\mathscr{F}_{\text {out }} & =\left\{f_{11}, f_{12}, f_{21}, f_{22}, f_{31}, f_{32}, f_{41}, f_{42}\right\}, \\
\mathscr{F}_{\text {in }} & =\left\{f_{01}\right\}, \\
\mathscr{P} & =\left\{\left\{f_{01}, f_{11}\right\},\left\{f_{01}, f_{21}\right\}\right\} \cup \mathscr{O} .
\end{aligned}
$$

\section{Enumeration of the case $\boldsymbol{n}=10$}

Equation (2) is written in the form

$$
F_{0}+F_{1}+F_{2}+F_{3}+F_{4}=14 \text {. }
$$

The lower bound condition is similar to the $n=9$ case. The solutions of (4) are $(1,3,3,4,3)$ and $(2,3,3,3,3)$. Enumeration of $(1,3,3,4,3)$ is similar to the case of $(1,3,3,3,3)$ for $n=9$ without the symmetry with respect to the $Y$-axis. We therefore consider the solution $(2,3,3,3,3)$ by the initial triangulation data shown in Fig. 5.

In this case, $f_{01}$ was fixed with the conditions $\left(f_{01} \nsim f_{11}\right) \wedge\left(f_{01} \nsim f_{21}\right),\left(f_{01} \nsim f_{21}\right) \wedge\left(f_{01} \nsim f_{31}\right)$, $\left(f_{01} \not f_{31}\right) \wedge\left(f_{01} \not f_{41}\right)$ and $\left(f_{01} \not f_{11}\right) \wedge\left(f_{01} \not f_{41}\right)$, followed by that for $f_{02}$ in a similar manner. By the multiplication principle, this gives 16 conditions. Because $(2,3,3,3,3)$ is symmetric with respect to the $X$-axis and $Y$-axis, and is diagonally symmetric, the condition is reduced to the following conditions:

(i) $\left(f_{01} \nsim f_{11}\right) \wedge\left(f_{01} \nsim f_{21}\right)$ and $\left(f_{02} \nsim f_{11}\right) \wedge\left(f_{02} \nsim\right.$ $\left.f_{21}\right)$

(ii) $\left(f_{01} \nsim f_{11}\right) \wedge\left(f_{01} \nsim f_{21}\right)$ and $\left(f_{02} \nsim f_{11}\right) \wedge\left(f_{02} \nsim\right.$ $\left.f_{41}\right)$,

(iii) $\left(f_{01} \nsim f_{11}\right) \wedge\left(f_{01} \nsim f_{21}\right)$ and $\left(f_{02} \nsim f_{31}\right) \wedge\left(f_{02} \nsim\right.$ $\left.f_{41}\right)$.

Thus we have to triangulate: $\mathscr{F}_{\text {out }}=$ $\left\{f_{11}, f_{12}, f_{21}, f_{22}, f_{31}, f_{32}, f_{41}, f_{42}\right\}, \mathscr{F}_{\text {in }}=\left\{f_{01}, f_{02}\right\}$, and a set $\mathscr{P}$ will be considered by the above condition individually.

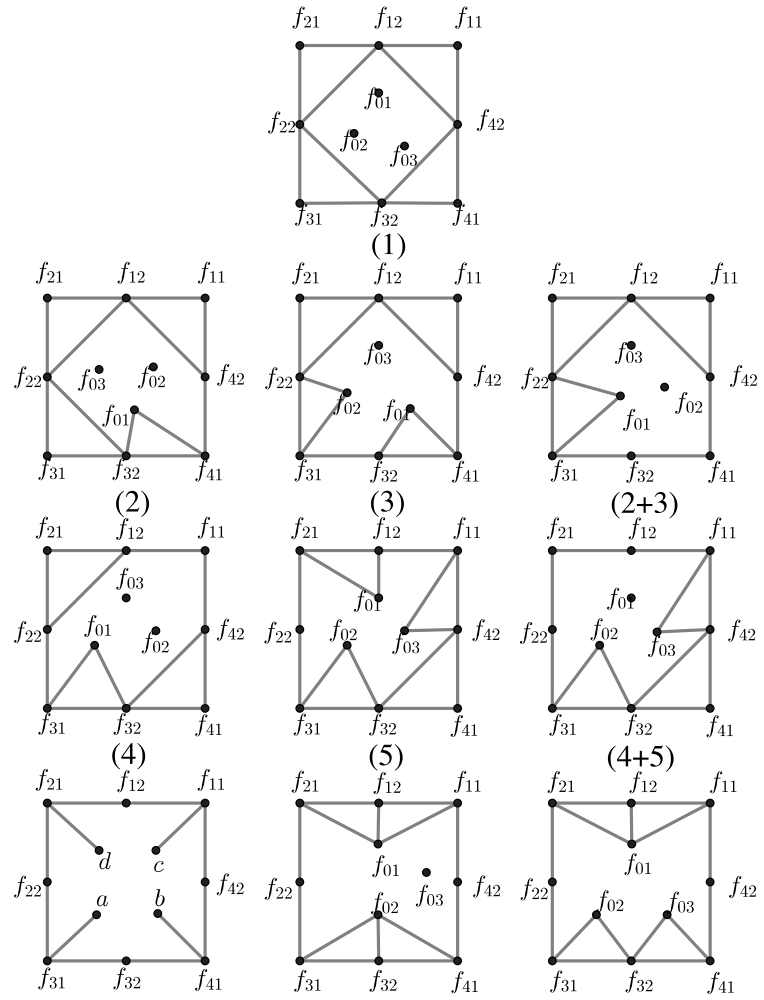

(6)
(6.2)

Fig. 6 Initial triangulating data for the solution $(3,3,3,3,3)$ when $n=11$.

\section{Enumeration of the case $n=11$}

Equation (2) is rewritten as

$$
F_{0}+F_{1}+F_{2}+F_{3}+F_{4}=15,
$$

with the conditions $F_{0} \geqslant 1$ and $F_{1}, F_{2}, F_{3}, F_{4} \geqslant 3$. The solutions of (5) are $(1,3,3,3,5),(1,3,3,4,4)$, $(1,3,4,3,4),(2,3,3,3,4)$ and $(3,3,3,3,3)$. The enumeration of the first four solutions are combinations of the case given above for $n=10$ without any symmetric cases. Hence we specify the solution for $(3,3,3,3,3)$.

If $F_{0} \geqslant 3$, the initial triangulating data will be larger. Hence we consider whether each corner is either a quadrilateral or an $n$-gon, where $n \geqslant 5$. If a combinatorial pattern $\pi$ consists of a quadrilateral at a corner, the dual combinatorial pattern $\pi^{*}$ consists of a triangle with the same corner. Along this part, we focus on the enumeration of the dual combinatorial pattern. The figures of dual combinatorial patterns are shown in Fig. 6 which indicates the initial triangulating data. The data in Fig. 6 are similar to the cases for $n=9$ and 10 and were hence generated by considering the following cases. 
Case 1: every corner of the combinatorial pattern is a quadrilateral. For a quadrilateral $Q$ at the corner of a combinatorial pattern $\pi$, there exists edges from two consecutive sides that are adjacent to $v_{0 k} \in \mathscr{V}_{0}$ for some $k$. Since $v_{0 k}$ is a vertex of degree 3 , there is an edge from $v_{0 k}$ that is laid outside $Q$. Hence the $f_{12} f_{22}, f_{22} f_{32}, f_{32} f_{42}$ and $f_{12} f_{42}$ edges exist in the dual graph. The initial triangulation data is shown in Fig. 6 in case 1 with $\mathscr{P}=\mathscr{O}$.

Case 2: only three corners of the combinatorial pattern are quadrilateral. In the dual graph, without loss of generality, suppose that $f_{32} \nsim f_{42}$. Thus a triangle $f_{42} f_{32} f_{41}$ does not exist and an edge from $f_{41}$ exists. If we consider the combinatorial pattern, the piece containing $f_{41}$ is consecutive to the piece containing $f_{32}$. From theorems 2 and 4 , there exists an internal piece consecutive to faces $f_{41}$ and $f_{32}$. That is, there is $f_{01} \in \mathscr{F}_{0}$ such that $f_{32} \sim f_{01} \sim f_{41}$. The initial triangulation data is shown in case 2 of Fig. 6 with $\mathscr{P}=\mathscr{O}$.

Case 3: only two consecutive corners of the combinatorial pattern are quadrilateral. Now, suppose that $f_{32} \not f_{42}$ and $f_{22} \not f_{32}$. We can consider case 2 together with case 3 by supposing that two consecutive corners are quadrilateral. To avoid overlapping with case 1 , we suppose that one corner is not quadrilateral and put no condition on the remaining corner. Without loss of generality, suppose that two upper corners are quadrilateral and the lower left corner is not a quadrilateral. The initial triangulation data is shown in the case $2+3$ of Fig. 6 with $\mathscr{P}=\mathscr{O}$.

Case 4: only two opposite corners of the combinatorial pattern are quadrilateral. Without loss of generality, suppose that the upper left and lower right corner are quadrilateral. Hence we have $f_{12} \sim$ $f_{22}$ and $f_{42} \sim f_{32}$. As with case 2 , suppose that there is $f_{01} \in \mathscr{F}_{0}$ such that $f_{31} \sim f_{01} \sim f_{32}$. We can leave the condition of the upper right corner and employ the condition $f_{12} \nsim f_{42}$.

Case 5: only one corner is quadrilateral in a combinatorial pattern. Suppose that there exists a triangle $f_{32} f_{41} f_{42}$ in a dual combinatorial pattern. As with case 2 , there exists $f_{01}, f_{02}, f_{03} \in \mathscr{F}_{0}$ such that $f_{12} \sim f_{01} \sim f_{21}, f_{31} \sim f_{02} \sim f_{32}$ and $f_{11} \sim$ $f_{03} \sim f_{42}$, which is shown in case 5 of Fig. 6 . In the dual combinatorial pattern, however, we can combine cases 4 and 5 by determining one corner as a triangle, omitting the occurrence of triangle in the two opposite corners, and put no condition on the remaining corner. The initial triangulation data is shown in case $4+5$ of Fig. 6 with $\mathscr{P}=$ $\left\{\left\{f_{02}, f_{11}\right\},\left\{f_{03}, f_{31}\right\}\right\} \cup \mathscr{O}$.
Case 6: every corner in a combinatorial pattern is not quadrilateral. Because we determined that each corner is not quadrilateral, there are edges from the corner point of the dual combinatorial pattern, i.e., face $f_{11}, f_{21}, f_{31}$, and $f_{41}$, as shown in case 6 of Fig. 6 . Define points $a, b, c$ and $d$ as in Fig. 6 case 6 . Since we have three interior points, there are two possible cases as follows.

Subcase $6.1 a$ and $b$ coincide, and $c$ and $d$ coincide. In subcase 6.1 of Fig. 6 , we denote the merged point from $a$ and $b$ as $f_{02}$ and the merged point from $c$ and $d$ as $f_{01}$. Let $f_{03}$ be the remaining internal vertex. Hence we triangulate the data shown in subcase 6.1 of Fig. 6 with $\mathscr{P}=$ $\left\{\left\{f_{01}, f_{31}\right\},\left\{f_{01}, f_{41}\right\},\left\{f_{02}, f_{11}\right\},\left\{f_{02}, f_{21}\right\}\right\}$.

Subcase 6.2: $c$ and $d$ coincide, but $a$ and $b$ do not coincide. Firstly, we show that the initial triangulation data in this case appears in the subcase 6.2 of Fig. 6, i.e., $a \sim f_{32}$ and $b \sim f_{32}$. Denote a vertex $f_{01}$ as with subcase 6.1 and let $a$ and $b$ be $f_{02}$ and $f_{03}$, respectively. It suffices to construct a triangle $f_{02} f_{31} f_{32}$ and $f_{03} f_{32} f_{41}$ by the following arguments. Note that the edge $f_{31} f_{32}$ must be contained in a triangle. From Theorem $4, f_{01}, f_{22}$, $f_{41}$ and $f_{42}$ may not be the vertex of this triangle. To ovoid overlapping with the previous subcase, $f_{02}$ must be the other vertex of that triangle. For a triangle with respect to $f_{32} f_{41}$, a similar argument was applied for the vertex $f_{03}$. Hence, in this case, we will triangulate with the initial triangulation data obtained in subcase 6.2 of Fig. 6 with $\mathscr{P}=$ $\left\{\left\{f_{01}, f_{31}\right\},\left\{f_{01}, f_{41}\right\},\left\{f_{02}, f_{11}\right\},\left\{f_{03}, f_{21}\right\}\right\}$.

\section{COMPUTATIONAL EXPERIMENTS}

We implemented the provided framework using Mathematica 9.0 with NMinimize, an optimization tool for finding the global minimum of a function. We let $d_{n}$ denote the optimal diameter shown in the original results ${ }^{5}$, and $D_{n}$ the optimal diameter obtained from our proposed algorithm. In the computations we used a default setting for which the working precision was equal to 16 . Note that the optimization in this work is convex programming.

In the case for $n=11$, we can generate 4642 distinct combinatorial patterns that satisfy our conditions. Fig. 7 shows the optimized results from the combinatorial patterns for each $n$. From the experiments, $d_{9}=0.454545, D_{9}=0.454545, d_{10}=$ $0.436467, D_{10}=0.436467$. In the case of $D_{11}$, the experimental result yields $D_{11}=0.416777$ such that $0.388730<D_{11}<0.416778$, which fits to the bound ${ }^{5}$. The results show that our results agree with their results in the cases of $n=9$ and 10, and 


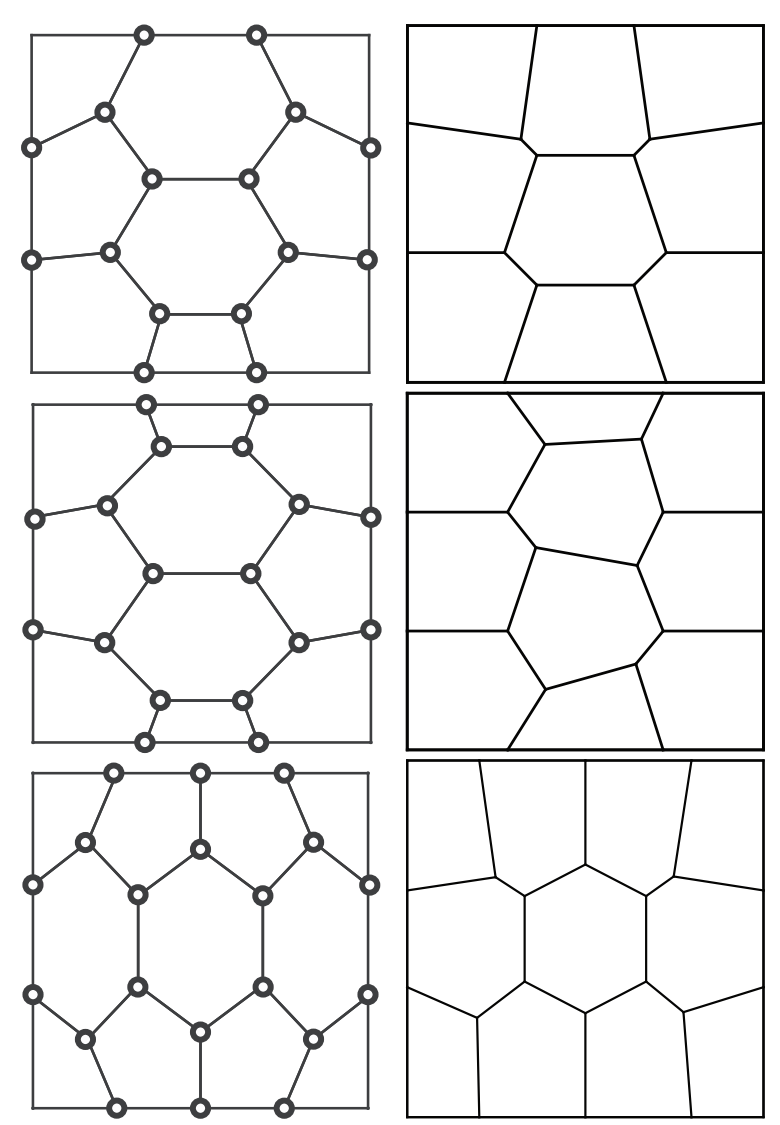

Fig. 7 The combinatorial pattern (left) and the optimized results (right). Results of $n=9$ (top), 10 (middle), 11 (bottom).

$D_{11}$ in this study also agrees with the prediction of Guy and Selfridge ${ }^{5}$.

Acknowledgements: The authors wish to thank the Development and Promotion of Science and Technology Talents Project by the Institute for the Promotion of Teaching Science and Technology, Ministry of Education, Thailand for the authors' scholarship. We also would like to thank R. K. Guy for his suggestion and for providing material of his work, and C. Likitvivatanavong for valuable comments and suggestions. This study is partially supported by the 90th Anniversary of Chulalongkorn University Fund (Ratchadaphiseksomphot Endowment Fund).

\section{REFERENCES}

1. Wenceslas GK, Lipman J (1958) Elementary problems and solutions: E1311. Amer Math Mon 65, 775.

2. Thompson GC, Munkres JR, Veech WA, Page Y, Goldstone LD (1959) Problems for solution: E1371-E1375. Amer Math Mon 66, 512-3.

3. Page Y, Selfridge JL (1960) Elementary problems and solutions: E1374. Amer Math Mon 67, 185.
4. Graham RL (1967) On partitions of an equilateral triangle. Can J Math 19, 394-409.

5. Guy RK, Selfridge JL (1973) Optimal covering of the square. Coll Math Soc János Bolyai 10, 745-99.

6. Jepsen $\mathrm{CH}$ (1986) Coloring points in the unit square. Amer Math Mon 17, 231-7.

7. Chaidee S, Wichiramala W (2013) Numerical approach to the optimal partitioning of a square problem. In: Proceedings of the AMM, Krabi, pp 199-206. 